\title{
INTRACRANIAL PRESSURE CHANGES DURING EXPERIMENTAL CONVULSIONS.
}

\author{
By MAXWELL E. MACDONALD AND STANLEY COBB, \\ Boston, Mass.*
}

The conspicuous symptom of epilepsy - the convulsion--has been studied from many angles, but little work has been done on the hydrodynamics of the spinal fluid during convulsions. This paper mentions a few clinical observations on the cerebrospinal fluid pressure during convulsions, and reports a short series of experiments on animals in which various pressure measurements were made during experimental convulsions. A series of fourteen animals were used for the intracranial pressure experiments, eight young dogs and six cats; and several rabbits were trephined for the direct observation of the cortex during a convulsion.

Thujone, the convulsive agent used, is the active principle of absinthe and is pharmacologically classified in the camphor group. The extract used in these experiments was kindly prepared by Professor Grinell Jones, of the Department of Chemistry of Harvard University, who distilled this preparation from oil of tansy. The exact point of its action is not definitely known, although Hildebrant, ${ }^{1}$ working on the camphor group of drugs in 1902, concluded that thujone acted on the medulla primarily and the vasomotor centre specifically. This is doubtful, because former experiments in this laboratory ${ }^{18}$ have shown that a much larger dose is needed to cause a convulsion in a decorticate or decerebrate animal than is needed to cause a similar convulsion in an intact animal, in which the cortex is active. In other words, we believe that the cortical motor cells are the ones which can be most easily stimulated by these convulsants.

It is sufficient for our purpose to say that the march of events during a convulsion produced by thujone is very similar to that seen during an epileptic fit. Within a few seconds after the drug is given intravenously the animal moves in a restless, uneasy manner and almost invariably raises its head and sniffs about the cage. Then one observes rapid constriction of the pupils and of the vessels of the ear. Immediately after

* From the Neuropathological Laboratory of the Harvard Medical School. This work was made possible by a generous grant from the Comnittee on Epilepsy, Charles I'. Howland, Secretary, 61 , Hroadivay, N.Y. 
this there is retraction of the head, tetanic extensor spasms of the fore limbs and more rarely of the hind limbs. Then comes the stage of clonic convulsion, during which the animal dashes and jerks about the cage. The actual convulsion is accompanied by dilatation of the pupils, exophthalmos, and marked congestion of the ear veins ; it is followed by a relaxed period with loss of sphincter control.

Our problem may be stated in two questions :-

1. What changes, if any, occur in the cerebrospinal fluid pressure during convulsions?

2. What are the relationships of these changes to blood pressure ?

There is still some discussion as to the exact relations between the intracranial venous, arterial and cerebrospinal fluid pressure. The generally accepted view at the present time appears to be that all sudden changes of intracranial pressure are parallel with venous pressure changes within the skull, which passively follow changes in the systemic circulation. Slow changes, such as those due to œdema, may not vary directly with intracranial venous pressure. This whole subject has been admirably reviewed by Weed. ${ }^{2}$

The question of vasomotor control of the cerebral vessels has not. been settled, nor has the possible influence of secretory activities been entirely ruled out. Physiological interpretation, therefore, will probably fare better at a later date.

\section{METHODS.}

In all cases 20 per cent. thujone in Norwegian cod-liver oil was given intravenously. The dosage for each animal was determined experimentally, as a variation in sensitivity to the drug has been found. In the average animal from 002 c.c. to 005 c.c. per kilogram of body weight produced a comparatively severe convulsion. Several times this dose is required to produce a similar convulsion in an animal under ether. In all operations ether was given by tracheal canula to insure a constant state of anæsthesia.

The animal was anæsthetized and the tracheal canula introduced. It was then placed in a ventral position and its legs extended and tied, while its head was held level by means of a jaw clamp. The jugular vein was exposed and connected to a manometer of $1 \mathrm{~mm}$. bore, partially filled with 4 per cent. sodium citrate solution. The saphenous vein was exposed for thujone injections and the femoral artery connected with a mercury manometer.

The skull was then exposed and a dentist's drill was used to make an opening about $2 \mathrm{~mm}$. in diameter. A lumbar puncture needle connected to a manometer of $1 \mathrm{~mm}$. bore, filled to a height of $110 \mathrm{~mm}$. with Ringer's solution, was introduced into the lateral ventricle. Readings were taken at regular intervals from the venous and cerebrospinal fluird 
manometers. The arterial pressure was recorded on a kymograph. After a control period thujone was introduced into the exposed vein, and the effect of the convulsion was observed. In four animals this procedure was modified by substituting a cisternal puncture for the ventricular puncture. In two animals the intracranial venous pressure was taken by a needle in the longitudinal sinus as described by Weed. ${ }^{2}$

The variation in cerebrospinal fluid pressure caused by gravity, as shown by Hill, ${ }^{3}$ has to be considered. In our experiments the animals were placed in a prone position and their heads were kept level. The intracranial pressures were considered as phenomena coming under the physical laws of the ' closed box,' the Monroe-Kellie doctrine. This has been restated by Weed and Hughson ${ }^{4}$ recently, and is now generally accepted. In performing ventricular punctures, therefore, an opening only large enough to admit the needle was made, and the needle was then sealed in place with wax. Care was taken to avoid loss of fluid. and to avoid the introduction of any considerable amount of fluid into the subarachnoid space through the manometer system.

\section{EXPERIMENTAL OBSERVATIONS.}

By these methods some twenty experiments were performed. Those that consisted of mere observation of the brain are described below. The manometer experiments are best explained by the accompanying charts, which show typical results.

A kymograph record of intracranial pressure was made by attaching a membrane tambour to a funnel-shaped glass sealed over a trephine hole. This record shows a distinct fall before the convulsion and a marked rise during the convulsion, indicating that the intracranial contents first shrink and then swell. Venous pressure records taken simultaneously show marked oscillations during the muscular spasms.

Direct observations of the brain were made in a number of animals, by exposing the cortex through a trephine hole. Thujone was given as in the other experiments. Just before a convulsion the brain appears to shrink and blanch. It retracts very definitely, leaving a space under the calvarium. That this stage is coincident with the fall in cerebrospinal fluid pressure was shown several times by observing the brain while a manometer was connected with the lateral ventricle. After the momentary blanching and shrinking, the brain surface becomes congested, seems to swell and pushes through the trephine opening. At this moment the fluid in the ventricular manometer rises to a high point.

It is interesting to note that the same sequence of events occurs in animals with lowered intracranial pressure following hypertonic salt solution. ${ }^{2}$ Observations were made on three such cases, and in each the 
CHAR'T 1.

Cat, N-23-50. Ventricular Puncture for C.S.F. Pressure, Jugular Vein for Venous Pressure, in mm. Ringer's Solution.

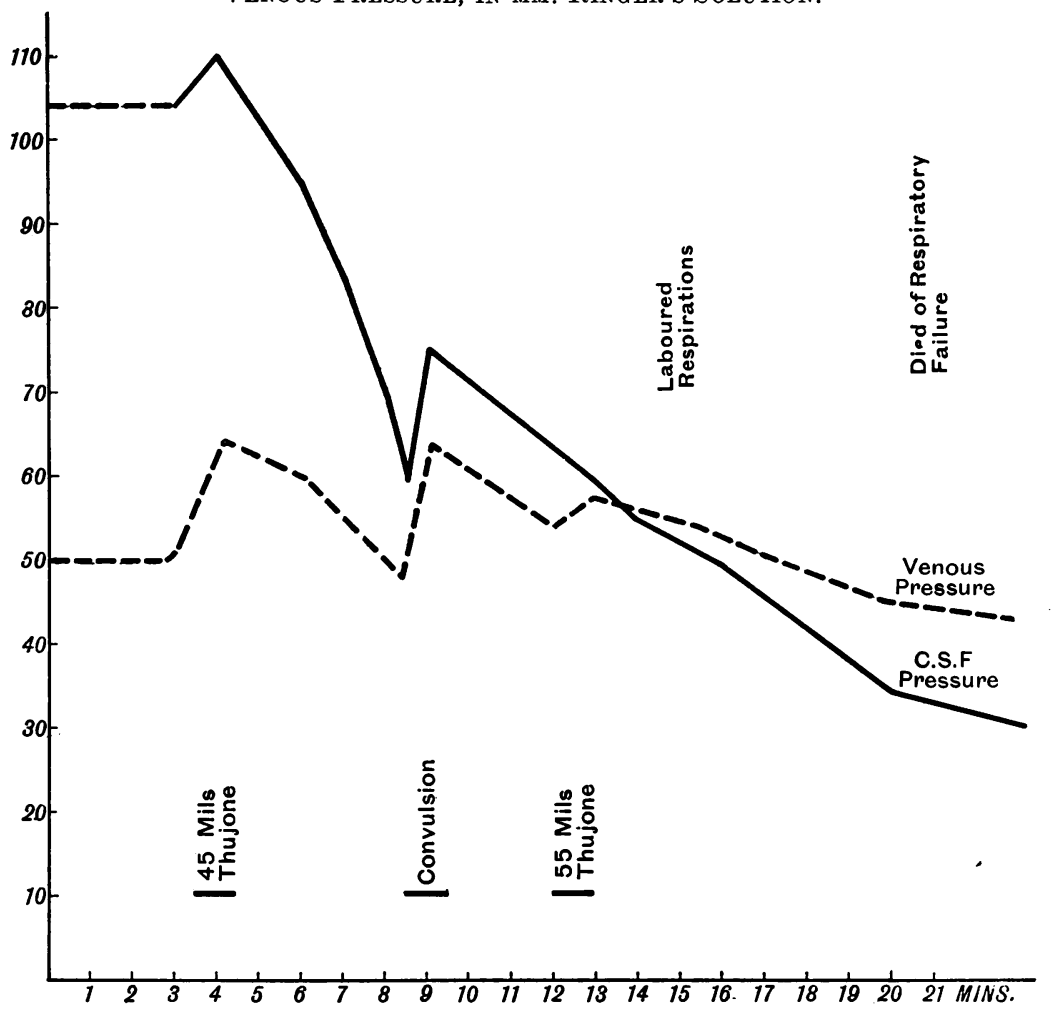

In this experiment the intracranial pressure is measured by ventricular puncture, the needle being connected with a manometer full of Ringer's solution. The venous pressure is simultaneously measured by a manometer connected with the jugular vein. 'The readings are in millimetres of Ringer's solution. It is seen that the intracranial pressure was $105 \mathrm{~mm}$. of Ringer's solution during the control period. During the injection of 0.45 c.c. of a 20 per cent. solution thujone, a slight rise occurred; then there was an immediate fall to $60 \mathrm{~mm}$., and, with the onset of the convulsion, a rise. The venous pressure is seen to keep quite parallel with the intracranial. In this case a second dose of thujone proved fatal.

subsequent rise was greater than that usually seen in the animals whose brains had not been previously shrunken with this hypertonic solution.

\section{CLINICAL CONSIDERATIONS.}

Three interesting clinical observations have been made recently during lumbar puncture on epileptics. In one case the operator had his needle in the lumbar subarachnoid space and was reading the pressure as registered by his manometer. Suddenly a marked fall in pressure occurred, so that he thought there must be a leak in the connections ; then there was a rapid rise of the fluid to the top of the manometer, over 
CHART 2.

Cat, N-23-47. C.S.F. Pressure viâ Cistern, in mm. Ringer's Solution.

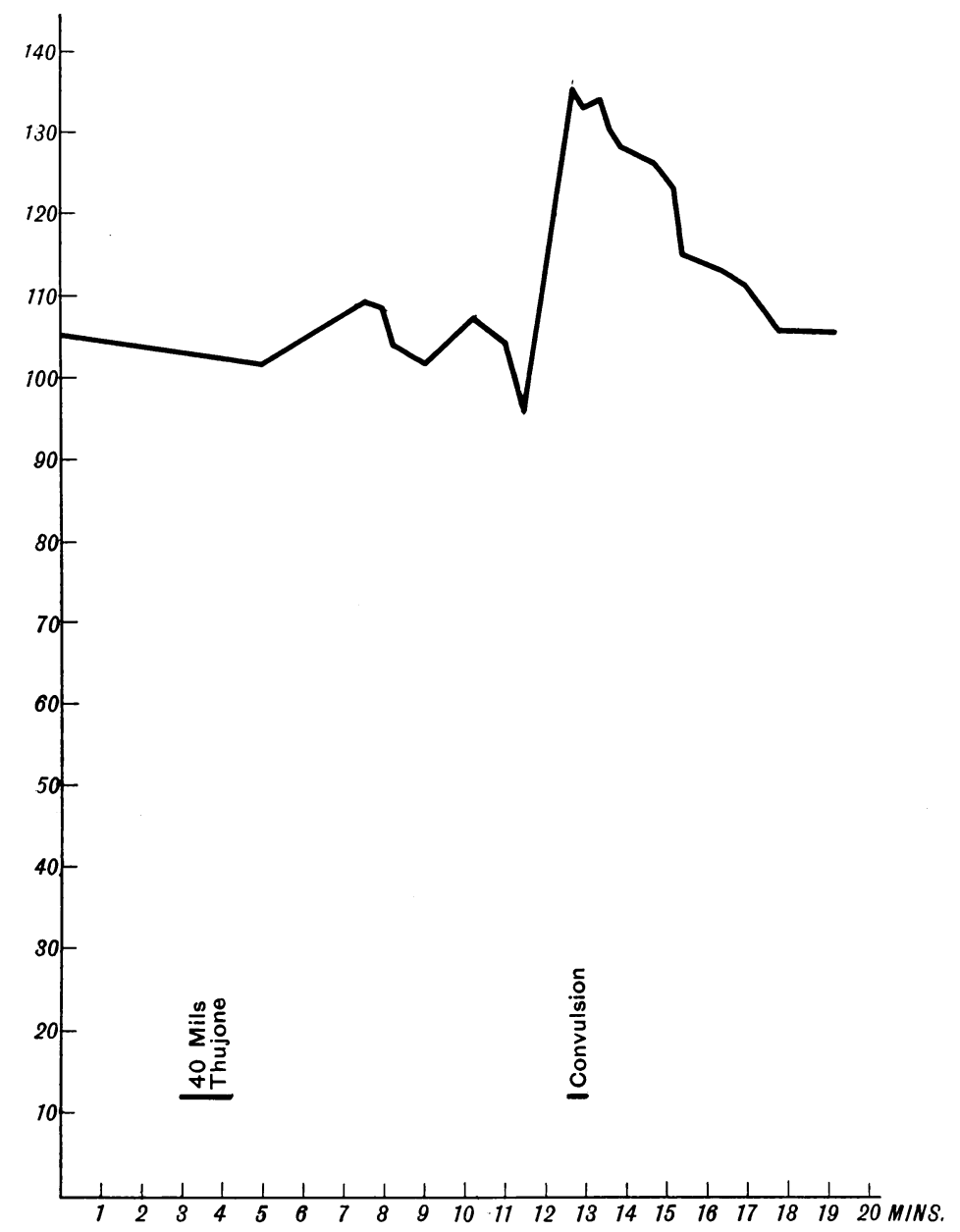

This experiment simply consists of recording the pressure of the cerebrospinal fluid by cistern puncture and a manometer filled with Ringer's solution. Immediately after the injection of thujone the preliminary fall in pressure is noted, but it is not as marked as is usually observed. The two small rises after the first drop correspond with slight twitching movements. Then there is a rather sudden drop before the onset of the severe convulsion. With the convulsion there is a conspicuous rise of pressure.

$400 \mathrm{~mm}$. It was then observed that the patient was having an attack, and the needle was removed.

In another case lumbar puncture was performed on a patient during status epilepticus; the fits were coming one after another at intervals of two to five minutes, and the patient was never really relaxed between them. The pressure was found to be high all the time, and during a hard 
convulsive period flowed out at the top of the manometer tube, that is, registered well above $400 \mathrm{~mm}$.

The third case was that of a paretic, who was being lumbarpunctured for treatment. Readings of the fluid pressure were being made, when suddenly a marked drop in pressure occurred. The needle

\section{CHART 3.}

Dog, N-23-51. Arterial Pressure, in mm. Mercury.

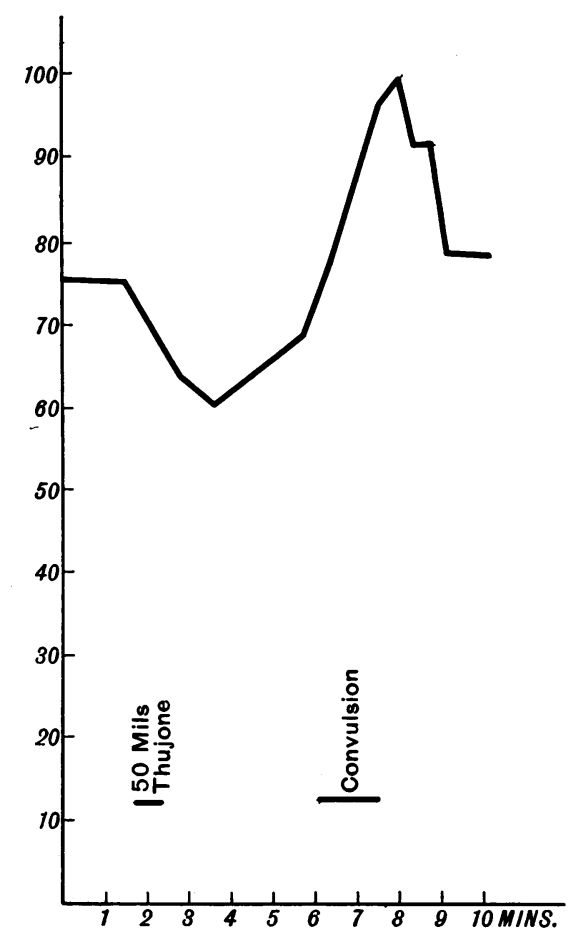

The curve is plotted from a kymograph record, to correspond with Charts 1 and 2, but the measurement is in millimetres of mercury. This curve is a typical one of the changes in arterial pressure during a convulsion. Following the thujone injection there is a drop and then a gradual rise before the onset of the convulsion. A sharp rise occurs as the muscular spasm of the fit supervenes.

was quickly removed, and a few seconds later the patient had a convulsion. Immediately after the convulsion the spinal fluid pressure was measured by another puncture, and was found to be back at its normal level. We wish to thank Drs. Mella, Mixter, and Solomon for allowing us to use their observations in these cases.

Leriche ${ }^{6}$ mentions that Virsin had seen the cerebrospinal fluid pressure double at the time of an epileptic crisis. Pollock ${ }^{7}$ has tracings 
of the blood pressure during a petit mal attack which show a preliminary rise, and then a sudden marked drop before an attack. The blood pressure remained low during the attack.

Quite a number of direct observations of the human cortex during an epileptic convulsion have been made by surgeons. Kennedy and Hartwell, ${ }^{8}$ watching the brain of a patient during a fit, noted, as the initial sign, a sudden whitening of the cortex, which was immediately replaced by a tremendous venous engorgement with protrusion of the brain beyond the level of the operative bone defect. Leriche ${ }^{6}$ noted in an operative case that the onset of a convulsion was preceded by the arrest of cerebral pulsation and blanching of the pial vessels. Horrax ${ }^{16}$ has frequently observed great bulging of the brain during convulsions. This experience of his was largely in military hospitals, while he was repairing hernias of the brain under local anæsthesia. He states that during the period of apnoa and rigidity the brain became congested and protruded through the skull defect, sometimes to such an extent that it was necessary for him to cover the wound with moist cotton and exert pressure with his hand in order to avoid destructive herniation. He remarks that similar, though less extensive, congestion and bulging may be observed by asking the conscious patient to cough, or strain or " bear down." Obviously these phenomena are the result of increased intrathoracic pressure. Thom ${ }^{9}$ has shown that dilated ventricles are a common post-mortem finding in epilepsy. Our own autopsy experience has corroborated this.

It occurred to us that dilatation of the cerebral ventricles might result mechanically from repeated convulsions and consequent high intraventricular pressure. Furthermore it is a frequent observation that sclerosis of the cornu ammonis is present in epilepsy. Steiner ${ }^{10}$ states that as many as 40 per cent. of epileptics show this lesion. Alzheimer ${ }^{11}$ says a little over one-half of cases of idiopathic epilepsy show it, about 60 per cent. of all cases of epilepsy being 'idiopathic.' Spielmyer, ${ }^{12}$ Kogerer, ${ }^{13}$ Wigglesworth and Watson, ${ }^{14}$ all note this sclerosis. Lynn-Thomas ${ }^{15}$ mentions a case of neoplasm with convulsions, dilated ventricles, and sclerosis of the cornu ammonis. Taft ${ }^{19}$ mentions changes of the same part in general paresis with convulsions. Such evidence leads one to speculate further. May it not be possible that the sclerosis of the cornu ammonis is a result of the dilatation of the ventricles-the end result of increased intraventricular pressure ?

With this point in view we studied several series of young rabbits, giving them frequent severe convulsions with thujone in an attempt to produce dilated ventricles and sclerosis of the cornu. Sixteen rabbits were used, but our results were negative. All the rabbits died young, four months being the longest period of survival. Most of them died of transverse myelitis, acquired during a fit. It is possible that less severe 
convulsions, continued over a longer period, might produce ventricular and hippocampal changes.

It may be that Dandy ${ }^{17}$ has the correct explanation of ventricular dilatation when he considers it a space-compensating function, the fluid replacing brain tissue in atrophic processes. The finding, however, of symmetrically dilated ventricles, with no obvious cerebral atrophy, would be hard to explain on this basis.

\section{SUMMARY.}

In a series of experiments it has been observed that during thujone convulsions there is an initial fall in the cerebrospinal fluid pressure just preceding the onset of the attack, and a rise in pressure during the fit. Coincident with the fall in pressure there is blanching and retraction of the cortex, followed by marked congestion and bulging. The cerebrospinal fluid pressure follows in general the curves of the peripheral venous and arterial pressures. There is some clinical evidence that similar changes take place during human epileptic convulsions.

\section{REFERENCES.}

1 Hildebrant, Archiv f. exp. Path. u. Pharm., 1902, xlviii, 151.

2 Weed, Physiological Rer., 1922, ii, 171.

3 Hilr, Leovard, "The Cerebral Circulation," London, 1896.

4 Weed and Hughson, Amer. Jour. Physiol., 1921, Iviii, 85.

5 Becht and Gunvar, Amer. Jour. Physiol., 1921, lvi, 231.

6 Leriche, Presse méd., 1920, xxviii, 615.

7 Pollock, Arch. Neurol. and Psychiat., 1923, ix, 608.

8 Kenveny, Arch. Neurol. and Psychiat., 1923, ix, 571.

9 Tном, Jour. Neri. Ment. Dis., 1920, li, 41.

10 Sterner, Arch. f. Psychiat., 1909-10, xlvi, 1091.

11 Alzheimer, quoted by Jeli.iffe and Whitf, "Diseases of the Nervous System," I'hila. and N.Y., 1915, 671.

12 Spielmyer, Münch. med. Woch., 1919, lxvi, 709.

13 Kogerer, Zeit. f. d. g. Neurol. u. Psychiat., 1920, lix, 116.

14 Wiggresworth and Watson, Brain, 1913-14, xxxvi, 31.

15 Iynn-Thomas, Brit. Jour. Surg., 1922, ix, 490.

16 Horrax, Personal communication.

17 Dandy, Johns Hopkins Hosp. Bull., 1923, xxxiv, 245.

18 Cobi and Uyematsu, Arch. Neurol. and Psychiat., 1922, vii, 660.

19 TAFT, Jour. Neurol. and Psychopath., 1921, ii, 221. 